\title{
Association between polymorphism of the cyclin E1 gene and susceptibility to hepatocellular carcinoma in Chinese Han population of Hubei
}

\author{
Qing Liu ${ }^{1}$, Jiayan Ling ${ }^{2}$, Qilong Song ${ }^{3}$, Xiaodong $\mathrm{Li}^{4}$, Chizhi Zhang ${ }^{4}$, Mingzhong Xiao ${ }^{4}$, Jie Huang ${ }^{1}$, Shuxia \\ $\mathrm{Han}^{5}$, Ling Liu ${ }^{5}$, Xiaoming Peng ${ }^{5}, \mathrm{Jie} \mathrm{Tan}^{5}, \mathrm{Yu} \mathrm{Wang}^{1}$, Li Ouyang ${ }^{1}$,Youyun Zhao ${ }^{5}$, Jianhua $\mathrm{Wu}^{5 *}$ \\ ${ }^{1}$ Department of Pathology,Hubei Provincial Hospital of TCM, Hubei Province Academy of TCM, Wuhan 430071,China; \\ ${ }^{2}$ Department of Traditional Chinese Medicine, the First Hospital of Wuhan, Wuhan 430022, China; \\ ${ }^{3}$ Department of Gastroenterology, the Central Hospital of Wuhan, Wuhan 430014, China; \\ ${ }^{4}$ Department of Infectious Disease, Hubei Provincial Hospital of TCM, Hubei Province Academy of TCM, Wuhan 430071, China; \\ ${ }^{5}$ Department of Laboratory, Hubei Provincial Hospital of TCM, Hubei Province Academy of TCM, Wuhan 430071, China.
}

\begin{abstract}
This study aimed to explore the relationship between CCNEl gene single nucleotide polymorphisms (SNP rs1406 and rs3218038) and the incidence of hepatitis B virus-related hepatocellular carcinoma (HCC) in the Chinese Han population in Hubei. A total of 663 subjects, including 173 HCC patients, $172 \mathrm{HBV}$-related liver cirrhosis (LC) patients, 162 asymptomatic HBV carriers (AsC), and 156 healthy controls, participated in the study. Genotyping of CCNE1 rs1406 and rs3218038 polymorphisms was done by illumina second generation sequence method.Our findings showed that $\mathrm{rs} 1406 \mathrm{G}>\mathrm{T}$ variant decreased the risk of $\mathrm{HCC}(\mathrm{OR} 0.710, P=0.035 \mathrm{G}$ vs T), and no significant differences were found between rs3218038 SNP and HCC risk using the $\chi^{2}$ test. Furthermore, stratified analysis revealed that differences in genotype frequencies were related to gender. Women who carried the CCNEl GT genotype were significantly associated with a decreased risk of HCC, compared with healthy controls carrying the GG genotype (additive model, OR 0.378,P=0.030). The results suggest that the rs $1406 \mathrm{G}$ allele and CCNEl rs1406 polymorphism produce an increased the risk of HCC in comparison with the T allele. Whereas, the GT genotype is a protective factor in the development of HCC in female patients.
\end{abstract}

Keywords: single nucleotide polymorphisms, hepatocellular carcinoma, second generation sequence

\section{INTRODUCTION}

Hepatocellular carcinoma (HCC) is a common malignancy and a leading cause of cancer death worldwide. An estimated 240 million people worldwide are chronically infected with the hepatitis B virus (HBV), placing them at increased risk of developing cirrhosis, hepatic decompensation, and hepatocellular carci- noma. Although most chronically HBV-infected subjects will not go on to develop hepatic complications, $15 \%-40 \%$ will give rise to serious sequelae during their lifetime ${ }^{[1,2]}$. The progression of HCC is a multiple process which is affected by multiple genetic and environmental factors such as alcoholism, aflatoxin or chemical carcinogens, hepatitis B or C viruses. Many factors contribute to HBV associated HCC, including

*Correspondence to: Jianhua Wu, Department of Laboratory, Hubei Provincial Hospital of TCM, Hubei Province Academy of TCM, No.4 Garden Hill, Yanzhi Road, Wuchang District, Wuhan 430071, Hubei Province, China. Tel: +86-27-88929236; Fax: +86-27-88929389; E-mail: wujianhuaemail@sina.com. 
HBV integration and mutation, and host susceptibility. Chronic HBV infection remains the major aetiological factor of HCC worldwide with more than one half of HCC patients being chronic carriers. In recent years, single nucleotide polymorphisms (SNPs) have been extensively studied in order to identify host genetic factors affecting HCC pathogenesis, and accumulated evidences in molecular genetics have indicated the correlation of genetic polymorphisms and HCC.

The protein encoded by the CCENl gene, belongs to the highly conserved cyclin family, which forms a complex and functions as a regulatory subunit of CDK2, whose activity is required for cell cycle G1/ $\mathrm{S}$ transition. The cell cycle is composed of a tightly regulated sequence of events whose main purpose is to ensure that genomic material is ready to be replicated, faithfully copied, and properly segregated into daughter cells. During HBV infection, viruses express proteins that perturb cellular DNA repair and cell cycle pathways, promoting tumorigenesis in their quest for cellular domination ${ }^{[3]}$. Viruses manipulate progression through the cell cycle and alter checkpoint signaling in order to provide a favorable environment for their own replication ${ }^{[4,5]}$. In so doing, they can predispose the infected cell to replicate DNA whose fidelity may be compromised, impairing thecell's ability to repair damaged DNA ${ }^{[6,7]}$, which together promote genomic instability. Alterations in the regulation of the cell cycle are strongly linked to tumorigenesis ${ }^{[4,8-10]}$, so genetic variants in genes critical to control of this cycle are good candidates to have their association with susceptibility to HCC assessed(Supplementary Table 1, visit the online version of APJBG at www.apjbg.com).

Recently, a human somatic cell reprogramming study demonstrated that a pluripotency specific spliced form of CCNEl specific to humans significantly enhanced reprogramming ${ }^{[11]}$. In addition, SNP expression analysis revealed that monoallelic gene expression was induced in the intermediate stages of reprogramming, while biallelic expression was recovered upon completion of reprogramming. Another study, using advanced sequencing technologies revealed that recurrent HBV DNA integration sites in hepatoma cells and the susceptible CCNEl gene played an important role in the pathogenesis of liver cancer ${ }^{[12]}$. The G/T single-nucleotide variation polymorphism locus rs1406 and rs3218038 on human chromosome 19 was obtained from Hapmap. Haploview software was used to conduct linkage disequilibrium and tagging algorithm.

Despite different studies revealing that polymorphisms in the CCNEl gene are associated with car- cinogenesis ${ }^{[13-15]}$, there has been limited data regarding the association of CCNEl SNP polymorphisms and HBV-related HCC. In the present study, we evaluated the relationship between CCNEl gene SNPs (rs1406 and rs3218038) polymorphisms and the incidence of HBV-related HCC.

\section{MATERIALS AND METHODS}

\section{Study subjects}

A total of 507 subjects including 173 HBV-related hepatocellular carcinoma (HCC) patients, $172 \mathrm{HBV}-$ related liver cirrhosis (LC) patients, and 162 asymptomatic HBV carriers (AsC) were recruited from the outpatient clinics and hospitalization wards at Hubei Provincial Hospital of TCM, Wuhan, China, between May 2012 and February 2016. The clinical criteria forHCC is defined byHBsAg and anti-HBcAb being positive for at least 6months, confirmed by biopsy, sonography, computed tomography or magnetic resonance imaging, with or without elevatedalphafetoprotein. The clinical criteria forLC is defined by patients being foundHBsAg and anti-HBcAb positive for at least 6months, with clinical presentation of gastroesophageal varices or a history of bleeding, ascites, edema, or encephalopathy, or serum albumin $<35 \mathrm{~g} / \mathrm{L}$, or total bilirubin $>35 \mu \mathrm{mol} / \mathrm{L}$, confirmed by biopsy, sonography, computed tomography, or magnetic resonance imaging, The clinical criteria forAsC is defined bythe presence of positive HBsAg over a period of six months, while their levels of alanine aminotransferase (ALT) remain within the normal range(Supplementary Table 2, visit the online version of APJBG at www. apjbg.com). One hundred and fifty-six healthy Han Chinese people were selected as controls from the Hubei Provincial TCM Hospital's health center. The criteria for healthy participants was that they had no previous diagnosis of liver disease, cancer or other serious illness and no family history of cancer, and were between the ages of 18 and 75 years old. The exclusion criteria were a current diagnosis and/or a history of cardiovascular disease, kidneydisease, severe metabolic disease, or psychiatric disease, pregnancy or lactation, allergic disease, and patients with HIV, HCV or other hepatotropic virus infection. Written informed consent was obtained from all study participants. The study met the criteria of the Hubei Provincial TCM Hospital's Institutional Review Board of Human Research.

Haploview software was used to screen the CCNEl gene and its role in the susceptibility to hepatocellular carcinoma, and CCNEl gene locations rs1406 and rs3218038 were obtained from the 
HapMap project.

\section{DNA extraction and genotyping of the $\mathrm{CC}-$ NE1SNPS}

Genomic DNA fromwhole blood was extracted from peripheral blood leukocytes using a TIANamp blood DNA kit (Tiangen Biotech [Beijing] Co. Ltd., Beijing, China). The concentration and purity of the DNA samples were detected by NanoDrop spectrophotometer. We excluded 25 samples that were genotyped unsuccessfully or were not in accordance with standard conditions $(1.6<$ DNA purity $<1.9)$. DNA samples were diluted to $4 \mathrm{ng} / \mathrm{L}$ and distributed in 96well plates (DNA panels), each of which contained 94 samples and 2 DNA-free control water. The analysis of CCNEl polymorphism rs1406 was performed by illumina second generation sequence method. The accuracy of the genotyping results was assessed using the ABI PRISM 3730 to examine the representative PCR-amplified DNA samples(Fig. 1and2). The SNP primer sequences and the necessary reaction conditions were listed in Table 1.

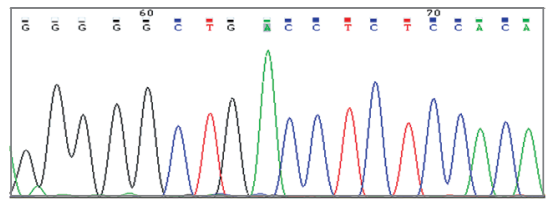

TT

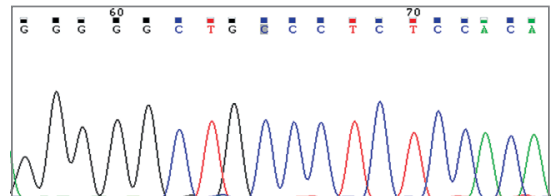

GG

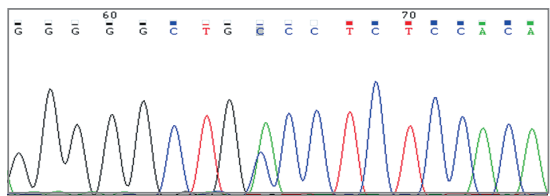

GT

Fig.1 rs1406 genotyping by direct sequencing. (a) TT genotype; (b) GG genotype; (c) GT genotype.

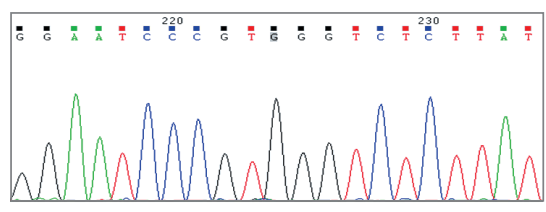

GG

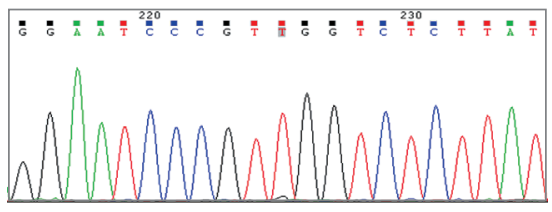

TT

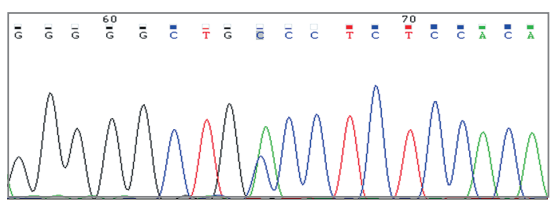

GT

Fig.2 rs3218038 genotyping by direct sequencing. (a) GG genotype; (b) TT genotype; (c)GT genotype.

Table 1 Primer sequence and the reaction condition for genotyping CCNE1 polymorphisms

\begin{tabular}{|c|c|c|c|}
\hline SNP & Primer sequence & Annealing temperature $\left({ }^{\circ} \mathrm{C}\right)$ & Product size (bp) \\
\hline \multirow[t]{4}{*}{ rs 1406} & F: $5^{\prime}-\mathrm{TCCAGCCTTGGTGACAGA} \mathrm{-} \mathrm{3'}$ & 50.7 & 79 bp \\
\hline & R: 5'-TCTCCTCGCAGGTGTTCT - $3^{\prime}$ & & \\
\hline & S : 5'-GGCATTGTACACTGTCAACTGAT - 3' & 52.8 & \\
\hline & A:5'-AGCGTTGTGCAGAGCCCATA - 3’' & & \\
\hline \multirow[t]{4}{*}{ Rs3218038 } & F: $5^{\prime}-$ AGCCATTGTGCCAAACTC $-3^{\prime}$ & 48.2 & $81 \mathrm{bp}$ \\
\hline & R: 5'-TTAGGGACCAGTGGATGA - $3^{\prime}$ & & \\
\hline & S: 5'-ATGAGGAGTTGGAGTGGTTAGG - 3' & 48.2 & \\
\hline & A: 5'-TACACCAAGTTTCAAAGCAAGAT - 3' & $54.8 \pm 22.5$ & \\
\hline
\end{tabular}

\section{Statistical analysis}

All statistical analyses were performed using SPSS software, version 17.0. The analysis of variance (ANOVA) method was used to evaluate the differences in demographic and clinical data among the four groups. To test for deviations from the Hardy-Weinberg equilibrium, the $\chi^{2}$ test was used to compare the true genotype frequencies in the study to the expected ones among the subjects. Binary logistic regression was used for calculating the relative risk of each SNP, controlling for age and gender as covariates. OR and their 95\% CIs were obtained as measures of association and precision between polymorphism genotypes. Dominant models were adopted for calculating odds ratios to assess the effect of an SNP variant. All statistical tests were two-sided and the statistical significance was set at $P<0.05$.

\section{RESULTS}

\section{Population characteristics}

Detailed patient demographics for all groups including gender, age, and HBV-DNA serum levels were listed in Table 2. The mean age of the healthy controls, asymptomatic HBV carrier group, HBVrelated liver cirrhosis group, and HBV-related HCC group were $46,44,55$, and 55 , respectively. In these retrospective analyses, we found that there was no significant difference in age between the healthy controls and AsC group $(P=0.172)$, however we observed a significant difference among the healthy controls and the LC and HCC groups. As for gender distribution from the subject profiles, we noted that there were higher numbers of men than women in each group. HBV-DNA serum levels did not dif- 
Table 2 Characteristics of study subjects

\begin{tabular}{lcccc}
\hline Groups & AsC & HCC & LC & Healthy controls \\
\hline No. of subjects & 162 & 173 & 172 & 156 \\
Gender & & & & 139 \\
Male, no, $(\%)$ & 120 & 126 & 33 & 87 \\
Female, no, $(\%)$ & 42 & 47 & $54.95 \pm 13.16$ & 69 \\
Age (years) $( \pm \mathrm{SD})$ & $43.95 \pm 14.85$ & $55.38 \pm 10.86$ & $4.72 \mathrm{E} 5 \pm 1.73 \mathrm{E} 6$ & $46.23 \pm 14.85$ \\
HBV-DNA (copies/mL) & $1.06 \mathrm{E} 7 \pm 3.24 \mathrm{E} 7$ & $6.66 \mathrm{E} 5 \pm 2.17 \mathrm{E} 6$ & No \\
P value & 0.172 & 0.000 & 0.000 & Ref \\
\hline
\end{tabular}

AsC, asymptomatic HBV carrier; HCC, HBV-related hepatocellular carcinoma; LC, HBV-related liver cirrhosis; HBV, hepatitis B virus; SD, standard deviation.

Supplementary Table 1. Diagnostic Criteria for AsC, ALF, LC, and HCC

\begin{tabular}{|c|}
\hline Asymptomatic $H B V$ carrier $(A s C)$ \\
\hline 1. $\mathrm{HBsAg}$ and anti-HBcAb positive for at least $6 \mathrm{mo}$ \\
\hline 2. Anti-HCV and HCV RNA negative \\
\hline 3. Anti-HDV and/or HDAg negative \\
\hline 4. Anti-HIV negative \\
\hline 5. ALT $<40$ and $\mathrm{AST}<40 \mathrm{IU} / \mathrm{L}$ \\
\hline 6. No clinical symptoms of hepatitis \\
\hline 7. No clinical liver cirrhosis \\
\hline 8. Age $\geqslant 35 \mathrm{y}$ \\
\hline Acute liver failure $(A L F)$ \\
\hline 1. $\mathrm{HBsAg}$ and anti-HBcAb positive for at least $6 \mathrm{mo}$ \\
\hline 2. Anti-HCV and HCV RNA negative \\
\hline 3. Anti-HDV and/or HDAg negative \\
\hline 4. Anti-HIV negative \\
\hline 5. Total bilirubin $>10$ times the upper limit of the normal range $(>171 \mu \mathrm{M} / \mathrm{L})$ \\
\hline 6. Prothrombin time activity $\leqslant 40 \%$ \\
\hline 7. Illness $<24$ wk duration \\
\hline 8. No clinical liver cirrhosis \\
\hline$H B V$-related liver cirrhosis $(L C)$ \\
\hline 1. $\mathrm{HBsAg}$ and anti-HBcAb positive for at least $6 \mathrm{mo}$ \\
\hline 2. Anti-HCV and HCV RNA positive \\
\hline 3. Anti-HDV and/or HDAg negative \\
\hline 4. Anti-HIV negative \\
\hline $\begin{array}{l}\text { 5. Liver cirrhosis with clinical presentation of gastroesophageal varices or a history of bleeding, ascites, edema, or encephalopathy, or serum albumin } \\
<35 \mathrm{~g} / \mathrm{L} \text {, or total bilirubin }>35 \mu \mathrm{mol} / \mathrm{L}\end{array}$ \\
\hline 6. LC confirmed by biopsy, sonography, computed tomography, or magnetic resonance imaging \\
\hline HBV-related primary hepatocellular carcinoma $(H C C)$ \\
\hline 1. $\mathrm{HBsAg}$ and anti-HBcAb positive for at least $6 \mathrm{mo}$ \\
\hline 2. Anti-HCV and HCV RNA positive \\
\hline 3. Anti-HDV and/or HDAg negative \\
\hline 4. Anti-HIV negative \\
\hline 5. HCC confirmed by biopsy, sonography, computed tomography, or magnetic resonance imaging, with or without elevated alpha-fetoprotein \\
\hline
\end{tabular}

HDV, hepatitis D virus; HDAg, hepatitis delta antigen; HBsAg, hepatitis B surface antigen; HBcAb, hepatitis B core antibody; HCV,hepatitis C virus; AST, aspartate aminotransferase; ALT, alanine aminotransferase; HIV, human immunodeficiency virus.

fer significantly between the HBV-related liver cirrhosis group and HBV-related HCC group $(P=0.25)$, whereas there was a significant difference among the asymptomatic HBV carrier group, LC group and HCC group $(P=0.02)$.

\section{AsC group and HBV-related LC group versus healthy controls}

The genotype and allele frequencies of CCNE1 gene polymorphisms among the AsC group and HBVrelated LC group and healthy controls were shown in Table 3. In the AsC group, the frequencies of GG, GT, and TT rs 1406 genotypes were $38.27 \%$, 48.76\%, and $12.96 \%$, respectively. While the frequencies of GG, GT, and TT rs3218038 genotypes were $61.73 \%$, $34.57 \%$, and $3.70 \%$, respectively. In the HBV-related LC group, the frequencies of GG, GA, and AA rs1406 genotypes were $39.53 \%, 48.25 \%$, and $12.21 \%$, respectively, and in the healthy controls, they were $36.53 \%$, $45.51 \%$, and $17.94 \%$, respectively. In the HBVrelated LC group, the frequencies of GG, GA, and AA rs3218038 genotypes were $56.40 \%, 38.95 \%$, and $4.65 \%$, respectively, and in the healthy controls, they were $62.18 \%, 35.26 \%$, and $2.56 \%$, respectively. No significant effects were observed between genotype and allele frequencies of the CCNEl gene rs1406 and 
Table 2 Supplementary Table 2. Characteristics of Variants Selected by DNA Pooling

\begin{tabular}{|c|c|c|c|c|c|c|c|c|}
\hline \multirow{2}{*}{ Affymetrix SNP ID } & \multirow{2}{*}{ SNP } & \multirow{2}{*}{ Gene } & \multirow{2}{*}{ Chr } & \multirow{2}{*}{ Location } & \multirow{2}{*}{ Allele } & \multicolumn{3}{|c|}{ Silhouette scores } \\
\hline & & & & & & ALF versus $\mathrm{AsC}^{\mathrm{a}}$ & LC versus $\mathrm{AsC}^{\mathrm{a}}$ & $\mathrm{HCC}$ versus $\mathrm{AsC}^{\mathrm{a}}$ \\
\hline SNP_A-1956120 & rs11866328 & GRIN2A & 16 & 9770057 & $\mathrm{~T} / \mathrm{G}$ & 0.48 & 0.71 & 0.84 \\
\hline SNP_A-2084283 & rs2013562 & UGT2B4 & 4 & 70389164 & $\mathrm{~A} / \mathrm{G}$ & 0.21 & 0.83 & 0.4 \\
\hline SNP_A-4213388 & rs7861010 & BNC2 & 9 & 16794082 & $\mathrm{~A} / \mathrm{G}$ & 0.27 & 0.8 & 0.72 \\
\hline SNP_A-4265842 & rs10485138 & ASCC3 & 6 & 101245311 & $\mathrm{~A} / \mathrm{G}$ & 0.66 & 0.51 & 0.8 \\
\hline SNP_A-1987038 & rs6909880 & ASCC3 & 6 & 101286075 & $\mathrm{G} / \mathrm{T}$ & 0.87 & 0.66 & 0.78 \\
\hline SNP_A-2031932 & rs10845858 & GRIN2B & 12 & 14045718 & $\mathrm{~A} / \mathrm{G}$ & 0.02 & 0.65 & 0.73 \\
\hline SNP_A-1970901 & rs1041236 & GPA33 & 1 & 165321604 & $\mathrm{~A} / \mathrm{G}$ & 0.51 & 0.58 & 0.71 \\
\hline
\end{tabular}

Silhouette scores: the Silhouette score was compared with AsC.

SNP, single nucleotide polymorphism; Chr, chromosome; Location, genomic position (NCBI Build 36); Allele, minor allele/major allele;ALF, acute liver failure; LC, liver cirrhosis; HCC, hepatocellular carcinoma; AsC, asymptomatic hepatitis B virus carrier.

rs3218038 polymorphisms using the $\chi^{2}$ test. SNP distribution was tested using the Hardy-Weinberg equilibrium (HWE) test, finding rs1406 and rs3218038 SNP distribution among the controls was consistent $(P=0.592, P=0.590$, respectively).

\section{HBV-related HCC patients versus healthy controls}

The genotype and allele frequencies of the CCNEl gene polymorphisms between HBV-related HCC patients and healthy controls are shown in Table 3. Using the $\chi^{2}$ test, we found that $\operatorname{rs} 1406 \mathrm{G}>\mathrm{T}$ variant decreased the risk of HCC (OR 0.710, 95\% CI 0.516$0.976, P=0.035 \mathrm{G} v s \mathrm{~T}$ ), but no significant differences between rs3218038 SNP and HCC risk (Table 3). Furthermore, we tested the Hardy-Weinberg equilibrium (HWE) for SNP, finding the distribution of rs 1406 SNP among the controls was consistent $(P=0.572)$.

\section{Stratified analysis}

We next investigated whether the differences in genotype and allele frequencies were related to gender. Significant differences in the distributions of CCNEl gene polymorphisms between HBV-related HCC patients and control groups were indeed observed (Table 4). Women who carried the CCNEl(rs1406) GT genotype (but not the TT genotype) were significantly related to a decreased risk of HCC, after adjusting for age using binary logistic regression analyses $(\mathrm{OR}=0.378,95 \%$ CI 0.157-0.910), compared with patients carrying the GG genotype $(P=0.030)$.

The frequencies of genotype and SNP rs1406 alleles in our control group were compared with those from the Haplotype Map (HapMap) Project (http:// www.ncbi.nlm.nih.gov/snp/). As seen in Table 5, we observed differences between polymorphisms in healthy controls in the present study and other ethnicities' healthy controls included in the HapMap project, namely allele frequencies in the CEU cohort (Utah residents with northern and western European
Table 3 Genotype and allele frequencies of two SNPs in the CCNE1 gene between HBV-related HCC patients and healthy controls

\begin{tabular}{lcccc}
\hline Groups & AsC & HCC & LC & $\begin{array}{c}\text { Healthy } \\
\text { controls }\end{array}$ \\
\hline rsl406 Genotype & & & & \\
G/G & $62(38.27)$ & $80(46.24)$ & $68(39.53)$ & $57(36.53)$ \\
G/T & $79(48.76)$ & $72(41.61)$ & $83(48.25)$ & $71(45.51)$ \\
T/T & $21(12.96)$ & $21(12.13)$ & $21(12.21)$ & $28(17.94)$ \\
Allele & & & & \\
G & $203(62.65)$ & $232(67.05)$ & $219(63.66)$ & $185(59.29)$ \\
T & $121(37.35)$ & $113(32.95)$ & $125(36.34)$ & $127(40.71)$ \\
\hline$P$ Value & 0.416 & 0.035 & 0.261 & Ref \\
\hline$P_{\text {HwE test }}$ & 0.592 & 0.572 & 0.444 & 0.475 \\
MAF & 0.373 & 0.363 & 0.329 & 0.407 \\
rs3218038 Genotype & & & \\
\hline G/G & $100(61.73)$ & $95(54.91)$ & $97(56.40)$ & $97(62.18)$ \\
\hline G/T & $56(34.57)$ & $71(41.04)$ & $67(38.95)$ & $55(35.26)$ \\
\hline T/T & $6(3.70)$ & $7(4.05)$ & $8(4.65)$ & $4(2.56)$ \\
\hline Allele & & & & \\
\hline G & $356(83.96)$ & $261(75.43)$ & $261(75.87)$ & $249(79.81)$ \\
\hline T & $68(16.04)$ & $85(24.57)$ & $83(24.13)$ & $63(20.19)$ \\
\hline$P$ Value* & 0.845 & 0.191 & 0.259 & Ref \\
\hline$P_{\text {HWE test }}$ & 0.590 & 0.158 & 0.402 & 0.241 \\
\hline MAF & 0.160 & 0.245 & 0.241 & 0.201 \\
\hline
\end{tabular}

"Comparisons are between Health controls and the other groups. Statistically signiticant values are shown in bold. HWE, Hardy-Weinberg equilibrium; MAF, minor allele frequency; AsC, asymptomatic HBV carrier; HCC, HBV-related hepatocellular carcinoma; LC, HBVrelated liver cirrhosis .

ancestry). The frequencies of genotype in JPT (Japanese in Tokyo) were also different from those in the present study. There was a lower detection rate of the GG allele $(29.06 \%)$ and a higher detection rate of the GT allele $(54.65 \%)$ at the rs 1406 site. However, there were no significant differences between SNPs in the present study and CHB (Chinese Han in Beijing).

\section{DISCUSSION}

HBV is a small enveloped DNA virus which primarily infects hepatocytes and causes persistent liver disease. Recent genetic studies indicated that HBV- 
Table 4 Stratified analysis of CCNE1 polymorphisms in healthy controls and HBV-related HCC patients

\begin{tabular}{|c|c|c|c|c|c|c|c|c|}
\hline & \multicolumn{4}{|c|}{ Female } & \multicolumn{4}{|c|}{ Male } \\
\hline & $\begin{array}{l}\text { Healthy controls } \\
n=69(\%)\end{array}$ & $\begin{array}{l}\mathrm{HCC} \\
n=47(\%)\end{array}$ & $\mathrm{OR}(95 \% \mathrm{CI})^{*}$ & $p$ Value & $\begin{array}{l}\text { Healthy controls } \\
n=87(\%)\end{array}$ & $\begin{array}{l}\text { HCC } \\
n=126(\%)\end{array}$ & $\mathrm{OR}(95 \% \mathrm{CI}) *$ & $p$ Value \\
\hline \multicolumn{9}{|c|}{ Genotypes } \\
\hline $\mathrm{G} / \mathrm{G}$ & 24 & 25 & 1 & & 33 & 55 & 1 & \\
\hline $\mathrm{G} / \mathrm{T}$ & 34 & 16 & $0.378(0.157,0.910)$ & 0.030 & 37 & 56 & $1.113(0.577,2.146)$ & 0.749 \\
\hline $\mathrm{T} / \mathrm{T}$ & 11 & 6 & $0.407(0.122,1.359)$ & 0.144 & 17 & 15 & $0.659(0.269,1.615)$ & 0.362 \\
\hline \multicolumn{9}{|c|}{ Allele } \\
\hline $\mathrm{G}$ & 82 & 66 & 1 & & 103 & 166 & 1 & \\
\hline $\mathrm{T}$ & 56 & 28 & $0.621(0.356,1.085)$ & 0.097 & 71 & 86 & $0.752(0.504,1.120)$ & 0.184 \\
\hline
\end{tabular}

"Logistic regression models were used for calculating the odds ratios (95\% confidential intervals) and corresponding $P$-values, controlling for age as covariates. Significant associations $(P<0.05)$ are in bold. HCC, HBV-related hepatocellular carcinoma; OR, odds ratio; CI, confidence interval.

Table 5 Comparison of genotype and allele frequencies in the healthy control subjects of the present study with examples from the HapMap project

\begin{tabular}{|c|c|c|c|c|c|c|c|c|}
\hline \multirow{2}{*}{$\begin{array}{l}\text { SNP } \\
\text { rs1406 }\end{array}$} & \multirow{2}{*}{$\begin{array}{c}\text { Samples } \\
\mathrm{N}\end{array}$} & \multicolumn{3}{|c|}{ Genotype frequency, $n(\%)$} & \multirow{2}{*}{$P$ values } & \multicolumn{2}{|c|}{ Alleles frequency, $n(\%)$} & \multirow{2}{*}{$P$ values } \\
\hline & & GG & GT & TT & & $\mathrm{G}$ & $\mathrm{T}$ & \\
\hline Prensent Study & 156 & $57(36.53)$ & $71(45.51)$ & $28(17.94)$ & REF & $185(59.29)$ & $127(40.71)$ & REF \\
\hline $\mathrm{HCB}$ & 86 & $32(37.20)$ & $40(46.51)$ & $14(16.27)$ & 1 & $104(60.46)$ & $68(39.53)$ & 0.847 \\
\hline $\mathrm{CHB}$ & 82 & $38(46.34)$ & $30(36.58)$ & $14(17.07)$ & 0.137 & $106(64.63)$ & $58(35.36)$ & 0.277 \\
\hline JPT & 172 & $50(29.06)$ & $94(54.65)$ & $28(16.27)$ & 0.107 & 194(56.39) & $150(43.60)$ & 0.477 \\
\hline YRI & 226 & $110(48.67)$ & $88(38.93)$ & $28(12.38)$ & 0.055 & $308(68.14)$ & 144(31.85) & 0.014 \\
\hline
\end{tabular}

HapMap, Haplotype Map; HCB, Chinese Han in Beijing, China; CHB, Chinese in Metropolitan Denver, Colorado; JPT, Japanese in Tokyo, Japan; CEU, Utah residents with northern and western European ancestry; and YRI, Yoruba in Ibadan, Nigeria.

related HCC displays a distinctive profile with a high rate of chromosomal alterations ${ }^{[16-17]}$. HBVs are streamlined organisms that lack many of the proteins required for genome replication, such as DNA polymerases. They rely on the host cell for these resources, which accumulate during the $S$ phase of the cell cycle to replicate cellular DNA. Viral activation of DDR pathways can lead to checkpoint signaling that stalls the cell cycle at Gl/S ${ }^{[18]}$. During an HBV infection, host cell cycle regulatory proteins and cyclin dependent kinase with different SNP sites are believed to have different roles in mediating cell DNA damage repair and gene instability.

The CCENl gene was found to associate with, and be involved in the phosphorylation of NPAT protein (nuclear protein mapped to the ATM locus), which participates in cell-cycle regulated histone gene expression, plays a critical role in promoting cell-cycle progression in the absence of pRB $5^{[19-20]}$ and forms a complex with CDK2, whose activity is required for cell cycle Gl/S transition. Activation of cyclin El(a key regulator of the Gl/S cell-cycle transition) has been implicated in many cancers including HCC. Although much is known about the regulation of cyclin El expression and stability, its post-transcriptional regulation mechanism remains incompletely understood since different SNP sites are likely to have different effects on mRNA expression and CCNEl gene viral integration risks. It was found that the cell cycle
CCNEl gene was one of the high frequency integration gene sites of the DNA virus. HBV and AAV2 are both DNA viruses associated with oncogenic insertional mutagenesis in human HCC. Nault JC et al. reported that AAV2 integrations occurred in the CCNEl gene, leading to an over expression of target genes ${ }^{[21]}$. Zhu BHet al. identified CCNEl integration sites in the host genome for the HBV-encoded X protein (HBx) in HCC biopsies that were positive for $\mathrm{HBsAg}^{[22]}$. Sung WK. et al. surveyed HBV integration in liver cancer genomes, identifying recurrent $\mathrm{HBV}$ integration events that were validated by RNA sequencing (RNA-seq) and Sanger sequencing at the known and putative cancer-related CCNEl genes ${ }^{[12]}$.

Alterations in the regulation of the cell cycle are strongly linked to tumorigenesis, so genetic variants in genes critical to control of the cycle are good candidates to have their associations to all kinds of cancer assessed. Many studies have indicated that the rs 1406 polymorphism in CCNEl gene is likely to contribute to an increased cancer risk. Amininia $\mathrm{S}$ et al. investigated the effects of single nucleotide polymorphisms in the CCNEl gene relating to the risk of breast cancer (BC) in an Iranian population in southeast Iran, concluding that the rs $1406 \mathrm{C} / \mathrm{A}$ polymorphism increased the risk of $\mathrm{BC}$ in codominant and dominant inheritance models ${ }^{[23]}$. Murali A et al. found that a significant risk of oral cancer was also evident for individual polymorphisms of cyclin E (at rs1406) ${ }^{[15]}$. 
Our findings showed that the rs1406 $\mathrm{G}>\mathrm{T}$ variant decreased the risk of HCC (OR $0.710,95 \%$ CI $0.516-$ $0.976, P=0.035 \mathrm{G}$ vs T) using the $\chi^{2}$ test. However, rs1406 SNP was not significantly associated with susceptibility to HCC after adjusting for age and sex using binary logistic regression analyses. Stratified analysis revealed that the differences in genotype frequencies were related to gender. Women who carried the CCNE1 GT genotype were significantly associated with a decreased risk of HCC compared with healthy controls carrying the GG genotype (additive model, OR 0.378, 95\% CI 0.157, 0.910, $P=0.030)$. In conclusion, this study indicated that the rs $1406 \mathrm{G}$ allele increased the risk of HCC in comparison with the $\mathrm{T}$ allele. The GT genotype was a protective factor in the development of HCC in female patients, and the CCNEl rs1406 polymorphism is likely to contribute to $\mathrm{HCC}$ risk.

\section{References}

[1] Ott JJ, Stevens GA, Groeger J, et al. Global epidemiology of hepatitis B virusinfection: new estimates of agespecific HBsAg seroprevalence and endemicity. Vaccine, 2012, 12(30): 2212-2219.

[2] Sarin SK, Kumar M, Lau GK,et al. Asian-Pacific clinical practice guidelines on the management of hepatitis $\mathrm{B}$ : a 2015 update. Hepatology International, 2016, 10(1): 1-98.

[3] Chaurushiya MS, Weitzman MD. Viral manipulation of DNA repair and cell cycle checkpoints. DNA Repair, 2009, 8(9): 1166-1176.

[4] Park US, Park SK, Lee YI, et al. Hepatitis B virus-X protein upregulates the expression of p21wafl/cipl and prolongs G1 $\rightarrow$ S transition via a p53-independent pathway in human hepatoma cells. Oncogene, 2000, 19(30): 3384-3394.

[5] O'Shea CC. Viruses-seeking and destroying the tumor program. Oncogene, 2005, 24(52): 7640-7655.

[6] Ransom M, Dennehey BK, Tyler JK. Chaperoning histones during DNA replicationand repair. Cell, 2010, 140(2): 183-195.

[7] Zender L, Villanueva A, Tovar V, et al. Cancer gene discovery in hepatocellular carcinoma. Journal of hepatology, 2010, 52(6): 921-929.

[8] ChoiYL, Park SH, JangJJ, et al. Expression of the G1-S modulators in hepatitis Bvirus-related hepatocellular carcinoma and dysplastic nodule: association of cyclin D1 and $\mathrm{p} 53$ proteins with the progression of hepatocellular carcinoma. Journal of Korean medical science, 2001, 16(4): 424-432.

[9] Kim H, Lee MJ, Kim MR, et al. Expression of cyclin D1, cyclin E, cdk4 and loss of heterozygosity of 8p, 13q, 17p in hepatocellular carcinoma: comparison study of childhood and adult hepatocellular carcinoma. Liver, 2000,
20(2): 173-178.

[10] Li Y, Yang XH, Fang SJ,et al. HOXA7 stimulates human hepatocellular carcinoma proliferation through cyclin E1/ CDK2. Oncology reports, 2015,33(2):990-996.

[11] Tanaka Y, Hysolli E, Su J, et al. Transcriptome Signature and Regulation in Human Somatic Cell Reprogramming. Stem cell reports, 2015,4(6):1125-1139.

[12] Sung WK, Zheng H, Li S, et al. Genome-wide survey of recurrent $\mathrm{HBV}$ integration in hepatocellular carcinoma. Nat Genet, 2012,44(7):765-769.

[13] Goode EL, Fridley BL, Vierkant RA,et al. Candidate gene analysis using imputed genotypes: cell cycle single-nucleotide polymorphisms and ovarian cancer risk. Cancer Epidemiol Biomarkers Prev, 2009, 18(3): 935-944.

[14] Han JY, Wang H, Xie YT, et al. Association of germline variation in CCNE1 and CDK2 with breast cancer risk, progression and survival among Chinese Han women. PLoS One, 2012, 7(11): e49296.

[15] Murali A, Nalinakumari KR, Thomas S, et al. Association of single nucleotide polymorphisms in cell cycle regulatory genes with oral cancer susceptibility. The British journal of oral \& maxillofacial surgery, 2014, 52(7): 652-658.

[16] Bonilla GR, Roberts LR. The role of hepatitis B virus integrations in the pathogenesis of human hepatocellular carcinoma. Journal of Hepatology, 2005,42(5):760-777.

[17] Zhai XL, Wang YJ, Chen P, et al. High CCNB2 expression correlates with poor prognosis in hepatocellular carcinoma. Asia-Pacific Journal of Blood Types and Genes, 2019, 3(1): 55-62.

[18] Hollingworth R, Grand JR. Modulation of DNA Damage and Repair Pathways by Human Tumour Viruses. Viruses, 2015, 7(5): 2542-2591.

[19] Skrajna A, Yang XC, Tarnowski K,et al. Mapping the Interaction Network of Key Proteins Involved in Histone mRNA Generation: A Hydrogen/Deuterium Exchange Study. Journal of molecular biology, 2016, 428(6): 1180-1196.

[20] Choi BH, Choi M, Jeon HY, et al. Hepatitis B viral X protein overcomes inhibition of E2Fl activity by $\mathrm{pRb}$ on the human $\mathrm{Rb}$ gene promoter. DNA Cell Biol, 2001, 20(2): 75-80.

[21] Nault JC, Datta S, Imbeaud S,et al. Recurrent AAV2related insertional mutagenesisin human hepatocellular carcinomas. Nat Genet, 2015, 47(10): 1187-1193.

[22] Zhu BH, Wang LT, Li T, et al. Identification of HBxrelated integration sites in HBsAg-positive hepatocellular carcinoma biopsy. Zhonghua gan zang bing za zhi(in Chinese), 2012, 20(6): 468-471.

[23] Amininia S, Hashemi M, Ebrahimi M,et al. Association between CCNE1 polymorphisms and the risk of breast cancer in a sample of southeast Iranian population. Medical oncology, 2014, 31(10): 189.

(Received 17 July 2019, Revised 14 October 2019, Accepted 14 November 2019) 